\title{
Should We Be Doing Implant-Based Breast Reconstruction in the Setting of Radiotherapy?
}

\author{
Grant W. Carlson, MD \\ Division of Plastic Surgery, Emory University, Atlanta, GA
}

The goals of postmastectomy reconstruction are to obtain a good aesthetic outcome and maintenance of quality of life (QOL) without negatively impacting cancer prognosis. Breast reconstruction is performed in less than $50 \%$ of patients treated by total mastectomy. ${ }^{1}$ The reason for this low utilization is multifactorial, and the decisionmaking process is complex. First and foremost is patient choice after an informed discussion with the treating physician. There is a great deal of concern on the part of surgeons that radiotherapy (RT) may be required postoperatively that could potentially compromise the reconstruction. This apprehension strongly influences the recommendations of the reconstructive surgeon. Implantbased reconstruction is the most common form of reconstruction, and its use has seen a marked rise in the last decade. $^{2}$ There is abundant data that radiation increases surgical morbidity after implant-based breast reconstruction. Barry and Kell in a meta-analysis of 11 studies found that patients receiving postmastectomy radiotherapy (PMRT) after implant reconstruction had significantly increased risk of complications (odds ratio [OR] 4.2; $95 \%$ CI 2.4-7.2) compared with those who did not receive radiation. ${ }^{3}$ Autologous reconstruction was associated with less morbidity (OR $0.21 ; 95 \%$ CI $0.1-0.4$ ) in the setting of PMRT. The impact of radiation therapy on implant breast reconstruction from the patients' perspective is less well documented.

Albornoz et al. ${ }^{4}$ performed a multicenter study that evaluated patient-reported outcomes in implant-based breast reconstruction with or without radiation using the

\footnotetext{
(C) Society of Surgical Oncology 2014
}

First Received: 5 January 2014;

Published Online: 18 April 2014

G. W. Carlson, MD

e-mail: grant_carlson@emory.org
Breast Q. The Breast $\mathrm{Q}$ is a validated patient-reported outcome measure (PROM) designed to quantify a patient's experience in terms of postsurgical satisfaction and healthrelated quality of life (QOL) after breast surgery and reconstruction. It can provide patients and providers with important information to assist in decision-making regarding breast reconstruction. The questionnaire was completed by 633 patients after implant reconstruction, 414 who did not receive radiation and 219 who received radiation (preoperative RT 47, postoperative RT 172). Patients who received postoperative RT had a significant reduction in all domains (satisfaction with breasts, satisfaction with outcome, psychosocial well-being, sexual well-being, and physical well-being) in univariate analysis. Patients who received radiation were younger and had higher rates of axillary dissection and chemotherapy. Multivariate analysis confirmed the negative impact of radiotherapy on "satisfaction with breasts" domain $(p=0.03)$ when adjusted for patient and treatment factors. The authors hypothesize that this may be a reflection of radiation side effects including capsular contracture and fibrosis. They conclude that the potential decrease in patient satisfaction after irradiation of implant reconstruction should be part of the informed decision making process.

These results confirm the findings of a similar study using the Breast $Q$ by Eriksson et al. ${ }^{5}$ They also found that RT significantly impacted all questionnaire domains when administered in the setting of implant reconstruction. These authors also reported the impact of RT on reconstructive failure: no RT $6 \%$, preoperative RT $25 \%$, and postoperative RT $15 \%(p<0.001)$. Patients with reconstructive failure were not included in the questionnaire analysis. Eriksson noted the large majority of women who were successfully reconstructed would choose breast reconstruction again regardless of whether they received radiation or not. They concluded that autologous reconstruction should be considered in the setting of RT, 
especially in previously irradiated patients. The reported high satisfaction rates showed that implant-based reconstruction is not contraindicated in the setting of radiotherapy.

There are several factors to be considered in analyzing the data presented by Albornoz et al. The study sites were high-volume specialty centers whose results in terms of patient selection and technical outcomes may not be applicable to lower-volume centers. Patients who knew they were to receive PMRT may have chosen not to have breast reconstruction. The timing to administering the satisfaction questionnaire is important. In the Albornoz study, the questionnaires were administered approximately 3 years after reconstruction. The aesthetic results of implant-based reconstruction tend to deteriorate with the passage of time. This is largely a function of native breast aging and progressive fibrosis around the breast implant. $\mathrm{Hu}$ et al. ${ }^{6}$ examined patient-reported satisfaction with breast reconstruction during long-term survivorship. They found that patient satisfaction with expander/implant reconstruction decreased with time. Patients more than 8 years post-reconstruction were significantly less satisfied compared with those who underwent implant reconstruction less than 5 years earlier. Importantly, reconstructive failures were not included in the PROM analysis by Albornoz. As reported by Eriksson, RT significantly increased the risk of reconstructive failure in implant reconstruction. ${ }^{5}$ Lam et al. ${ }^{7}$ reviewed 12 studies involving 1,853 patients to examine the impact of PMRT on immediate 2-stage implant reconstruction. Post-reconstruction radiotherapy resulted in a significantly higher reconstructive failure compared with controls (18.6 vs $3.1 \%$, $p<0.00001$ ). Reconstructive failure could have a greater negative impact of patient satisfaction and QOL than mastectomy without attempted reconstruction. Many of these patients will also undergo secondary reconstruction with further potential for morbidity and decreased PROMs.

Chest wall irradiation negatively impacts all aspects of implant-based breast reconstruction. There is abundant level III evidence that RT increases surgical complications and reconstructive failure. Albornoz and her coauthors provide important information about its deleterious effects on patient satisfaction and quality of life. In total, this information is valuable to enable a patient to make an informed decision regarding implant-based reconstruction in the setting of radiation.

Should we be doing implant-based breast reconstruction in the setting of radiotherapy? The answer is yes. The glass, though, is clearly only half full. Many patients are not candidates or do not desire autologous breast reconstruction. The majority of these patients can be successfully reconstructed with breast implants in this setting. That being said, patients need to be counseled regarding the negative impacts of radiotherapy.

\section{REFERENCES}

1. Sisco M, Du H, Warner JP, Howard MA, Winchester DP, Yao K. Have we expanded the equitable delivery of postmastectomy breast reconstruction in the new millennium? Evidence from the national cancer data base. J Am Coll Surg. 2012;215:658-66; discussion 666.

2. Albornoz CR, Bach PB, Mehrara BJ, Disa JJ, Pusic AL, McCarthy $\mathrm{CM}$, et al. A paradigm shift in U.S. Breast reconstruction: increasing implant rates. Plast Reconstr Surg. 2013;131:15-23.

3. Barry M, Kell MR. Radiotherapy and breast reconstruction: a meta-analysis. Breast Cancer Res Treat. 2011;127:15-22.

4. Albornoz C, Matros E, McCarthy C, et al. Implant based reconstruction and radiation: a multicenter analysis of long-term health-related quality of life and satisfaction. Ann Surg Oncol. 2014. doi:10.1245/s10434-014-3483-2.

5. Eriksson M, Anveden L, Celebioglu F, Dahlberg K, Meldahl I, Lagergren $\mathrm{J}$, et al. Radiotherapy in implant-based immediate breast reconstruction: risk factors, surgical outcomes, and patientreported outcome measures in a large Swedish multicenter cohort. Breast Cancer Res Treat. 2013;142:591-601.

6. Hu ES, Pusic AL, Waljee JF, Kuhn L, Hawley ST, Wilkins E, et al. Patient-reported aesthetic satisfaction with breast reconstruction during the long-term survivorship period. Plast Reconstr Surg. 2009;124:1-8.

7. Lam TC, Hsieh F, Boyages J. The effects of postmastectomy adjuvant radiotherapy on immediate two-stage prosthetic breast reconstruction: a systematic review. Plast Reconstr Surg. 2013;132:511-8. 